\title{
Proton Synchrotron RF Cavity Mode Damper Tests
}

\author{
W. R. Smythe \\ University of Colorado \\ Boulder, CO 80309-0446 \\ C. C. Friedrichs and L. S. Walling \\ AT Division, Los Alamos National Laboratory \\ Los Alamos, NM 87545
}

\section{Abstract}

A versatile, broad frequency range, rf cavity mode damper has been described in a previous paper. ${ }^{1}$ A mode damper of this type has now been designed, constructed, installed, and tested on an existing $50 \mathrm{MHz}$ synchrotron cavity. Measurements on the combined system have been completed, affording a comparison of analytical predictions with actual performance. The results of the measurements confirm the predicted efficacy of the mode damper up to $1000 \mathrm{MHz}$.

\section{INTRODUCTION}

The mode damper cavity illustrated in Figure 1 was designed for installation on the existing Los Alamos main ring cavity, ${ }^{2}$ a $50 \mathrm{MHz} 150 \mathrm{kV}$ prototype synchrotron cavity employing a perpendicularly biased ferrite tuner. Subsequently, both the prototype cavity and the mode damper were moved to the Superconducting Super Collider Laboratory near Dallas, Texas, where these measurements were performed.

The basic idea of the mode damper is to load down the high order cavity modes heavily without loading down the fundamental (accelerating) mode any more than is tolerable. The important elements in the mode damper circuit are: the capacity of the accelerating gap, the mode damper cavity, and the damping resistance which shunts the entrance to the damper cavity. In the low frequency regime, the equivalent damping resistance at the gap decreases approximately as the inverse fourth power of the frequency. This can be seen by considering that the current across the accelerating gap capacitance, at fixed voltage, is proportional to the frequency. The reactance which the (inductive) damper cavity presents to this current is also proportional to the frequency, so the power dissipated in the damping resistor is proportional to the fourth power of the frequency in the low frequency limit.

\section{Analytical PREdictions}

Two methods of analytically predicting the performance of the mode damper have been employed. The first of these has been described in detail elsewhere, ${ }^{1}$ and will be only sketched here. The first method uses the computer program SUPERFISH ${ }^{3}$ to calculate three parameters of the slot type damper cavity: the frequency, $f_{1}$, at which the impedance at the entrance slot is a maximum, $(250 \mathrm{MHz}$ in this case); the corresponding $\mathrm{R} / \mathrm{Q}$ of the cavity (18.6 ohms); and the frequency, $\mathrm{f}_{2}$, at which the impedance at the entrance slot is a minimum, effectively shorting out the damping resistor (1124 MHz). The damper cavity is then represented by an analytical model which consists of two sections of coaxial line of different lengths and impedances, ending in a short circuit. The line parameters are adjusted to reproduce $f_{1}, R / Q$, and $f_{2}$. This model, together with values for the accelerating gap capacitance and the damping resistance, permit the calculation of the effective damping resistance appearing across the accelerating gap as a function of frequency. The results of this calculation are shown as the smooth curve in Figure 5.

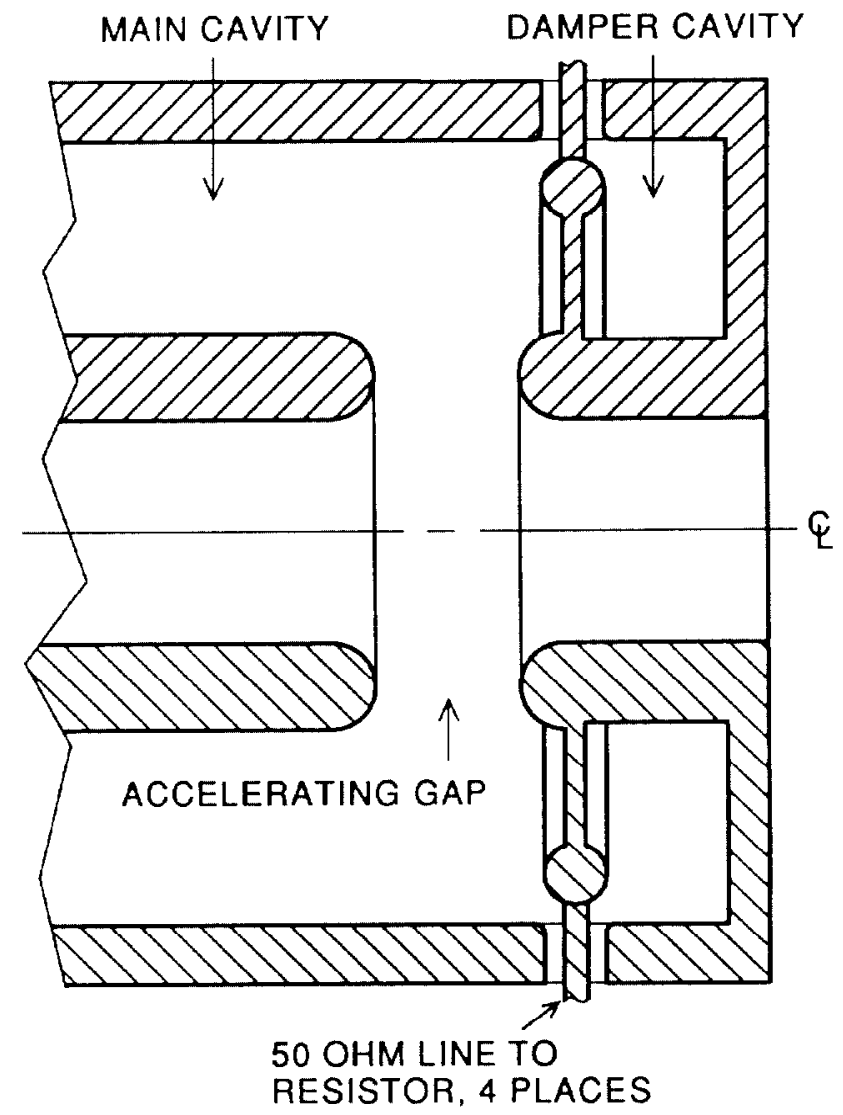

Fig. 1. Cross section drawing of the mode damper. The four $50 \Omega, 1 \frac{5}{8}$ " coaxial lines located $90^{\circ}$ apart are connected to rf loads. 
The second analytical method of predicting the performance of the mode damper employs SUPERFISH to model the accelerating gap and the damper cavity (without the damping resistors, of course). The rest of the cavity is simply replaced by a coaxial line of adjustable length. The ficlds at the gap and at the damping resistor are found at various frequencies by letting SUPERFISH solve the problem for various short positions. Figures 2 and 3 show field configurations
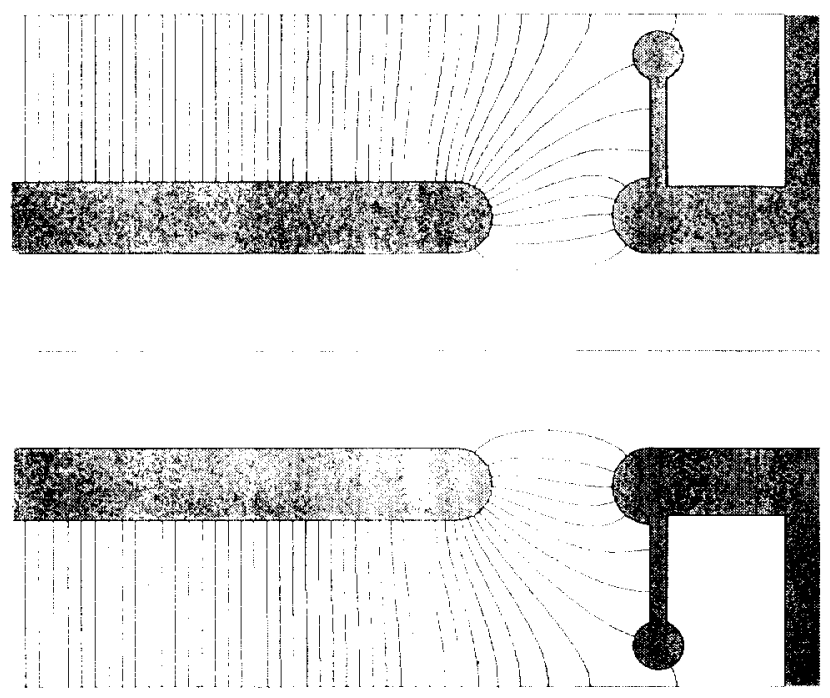

Fig. 2. SUPERFISH electric field plot at the fundamental frequency, $50 \mathrm{MHz}$. Very little electric field is developed across the damper cavity at this frequency.
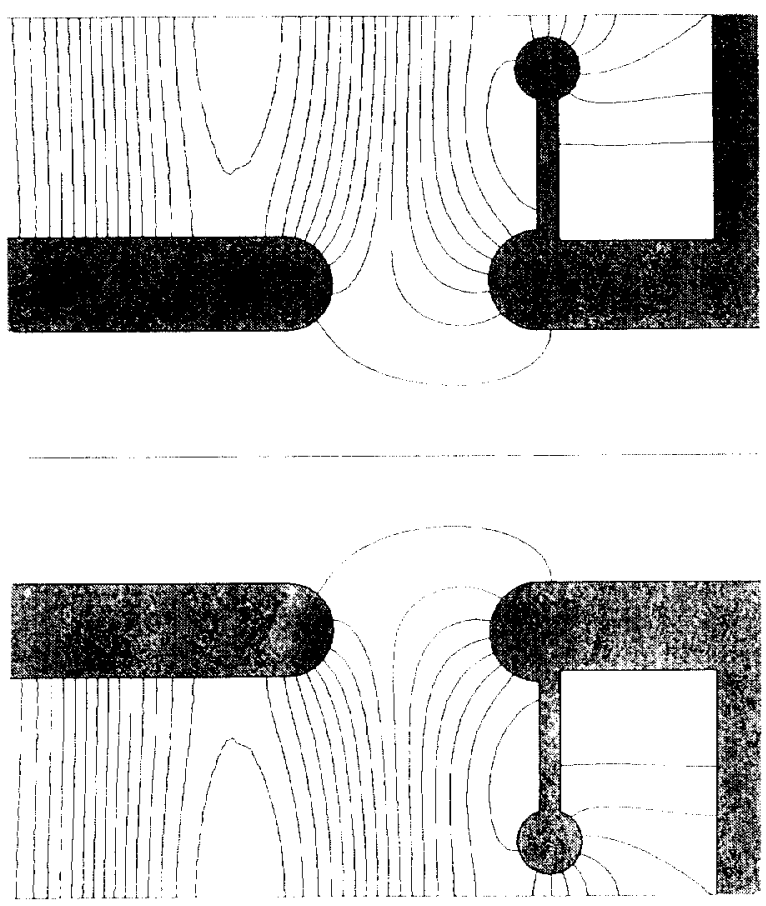

Fig. 3. SUPERFISH electric field plot at 964 MHz. Large fields are developed across the damper cavity. It is clear that a lumped constant representation of the gap capacitance is not valid. calculated by SUPERFISH. Use of the SUPERFISH post processors SF01 and SHY allows the voltage across the damping resistor and the voltage across the gap to be determined. The value of the damping resistor is multiplied by the square of the gap/damper voltage ratio to obtain the equivalent damping resistance at the gap. This method is expected to give good results under most conditions; however, some cautions must be noted. The method is not valid if the current drawn by the resistor beromes comparable to, or larger than, the current present on the cavity structure at the point of attachment. It should also be remembered that the actual damping resistor consists of two 50 ohm resistors (or sometimes four $100 \mathrm{ohm}$ resistors), so there is some inexactness in the assumed azimuthially symmetric distribution of the current. As long as the resistor current is a small fraction of the structure current, the calculation is expected to give reasonable results. The points in Figure 5 indicated by crosses were calculated in this way. Inspection of the SUPERFISH field plots at higher frequencies, Figure 3 for example, indicates that the field patterns do show wave length effects, and that the lumped constant model for gap capacitance is inadequate at high frequency. Thus, at these higher frequencies it is expected that the SUPERFISH calculation method will give more accurate results. It is pleasing that the SUPERFISH calculations predict better performance of the mode damper in the high frequency region.

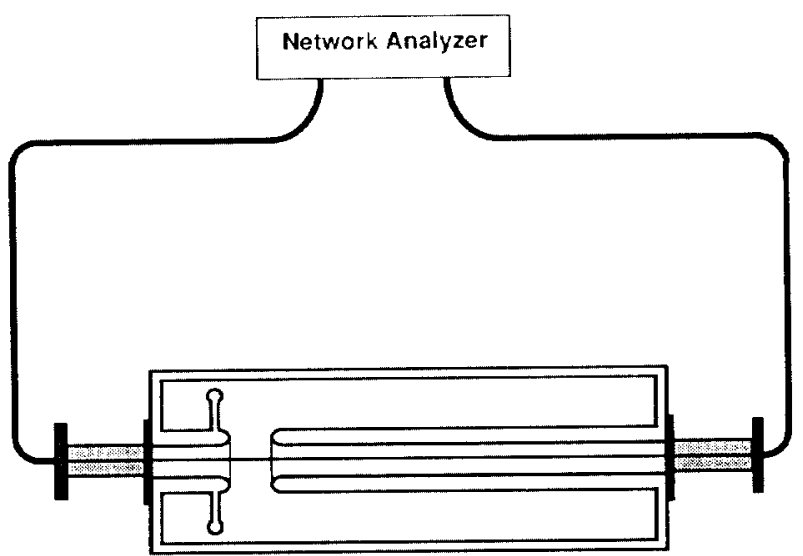

Fig. 4. Shunt impedance measurement arrangement. The cavity impedance at the gap appears in series with the outer conductor of the coaxial line connecting the two ports of the network analyzer.

\section{Test Measuremen't's}

The measurement scheme employed was designed to measure as directly as possible the (longitudinal) impedance seen by the beam current as it crosses the accelerating gap. The arrangement is illustrated in Figure 4. A Hewlett-Packard model $8753 \mathrm{C}$ network analyzer was employed. The cavity beam pipe is made into a coaxial line by the insertion of a $3 \mathrm{~mm}$ diameter 
brass rod on its axis. The network analyzer is connected to this line by type RG 141 coaxial cable and a pair of adapters. Thus, the cavity gap appears as a break in the outer conductor of the coaxial line connected between two ports of the network analyzer. The system is calibrated by replacing the cavity successivcly by a short line, a longer line, and by a short circuit. Use and calibration of this type of measuring system have been previously described. ${ }^{4}$ The calibration corrects for the effects of the line discontinuities and allows the determination of the impedance appearing at the gap, as a function of frequency.

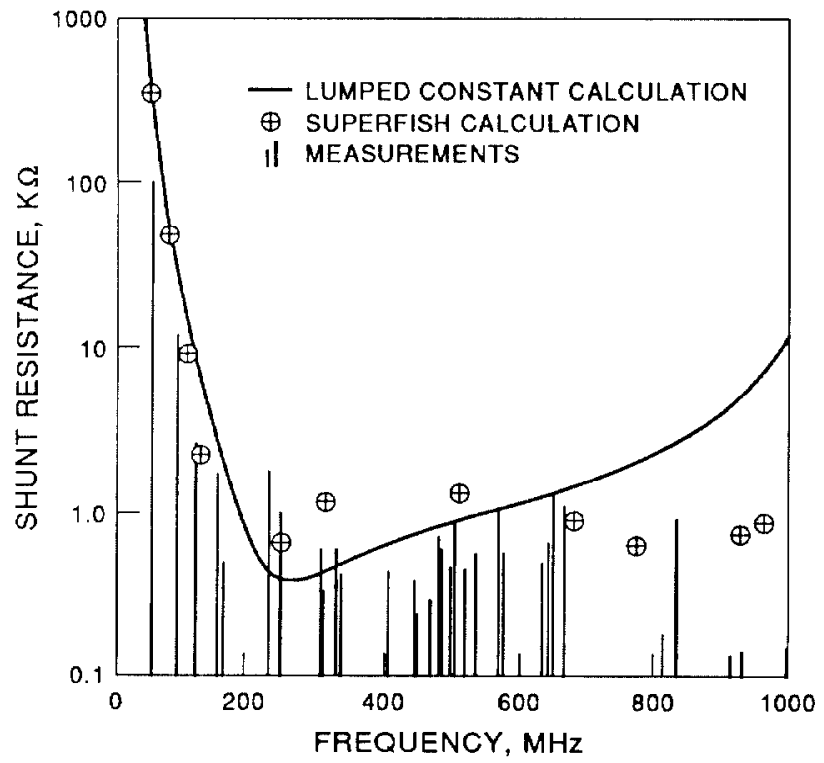

Fig. 5. The equivalent damping resistance of the mode damper as a function of frequency. The measured points are only at cavity higher order mode frequencies. Their measured shunt impedance is the equivalent damping resistance reduced by the natural shunt resistance of the mode.

\section{Results and Conclusions}

The results of the impedance measurements with four $100 \mathrm{ohm}$, five $\mathrm{kW}$ damping resistors connected are shown in Figure 5 as vertical lines. Each resistor was connected to the damper cavity by $20 \mathrm{~cm}$ of 50 $\Omega$ line. Each line represents a mode in the cavity at that frequency. The line extends up to the value of shunt resistance measured for that mode. The measured shunt impedance of a resonance is the combination of the natural shunt impedance of that resonance combined in parallel with the effective damping resistance at that frequency, so the measured values should all be below, or at most equal to, the effective damping resistance.

Inspection of the results presented in Figure 5 allows us to draw several conclusions. First, the mode damper appears to behave as predicted up to $1000 \mathrm{MHz}$. Second, it appears that SUPERFISH calculations are the better predictor of mode damper performance, as expected.

The damping of the fundamental mode is of major interest. The lumped constant method predicted a damping resistance of $367 \mathrm{k} \Omega$ at $50.0 \mathrm{MHz}$, while the SUPERFISH method predicted a damping resistance of $358 \mathrm{k} \Omega$ at $49.8 \mathrm{MIIz}$. Experimental measurement of the corresponding case with damping resistors on and off gave a mcasurcd value for the effective damping resistance at the fundamental mode, $51.0 \mathrm{MHz}$, of 312 $\mathrm{k} \Omega$, which corresponds to $342 \mathrm{k} \Omega$ when corrected to $49.8 \mathrm{MHz}$ by the frequency to the inverse fourth power law. Thus, the actual loading of the fundamental mode appears to be in good agreement with both calculations.

It appears that the overall performance of this mode damper can be adequately predicted analytically. It also appears to damp all significant, higher order modes. It is quite rugged, reasonably compact, and adaptable to a variety of cavities. Further, the damping can be reduced or increased after installation, depending on the needs of the accelerator.

\section{ACKNOWLEDGEMENTS}

We wish to thank Dale Colcman, Gilmer Ilulsey, and Fred Brandeberry of the Superconducting Super Collider Laboratory for the valuable assistance which they provided with the planning and execution of the measurements at the SSCL. In addition, we gratefully acknowledge valuable discussions with Dale Coleman regarding the possibility of higher order modes in the damper cavity.

\section{REFERENCES}

[1] W. R. Smythe, T. A. Enegren, and R. L. Poirier, "A Versatile RF Cavity Mode Damper," in Proceedings of the 2nd European Particle Accelerator Conference, Edited by P. Marin and P. Mandrillon, Editions Frontiers, Gif-Sur-Yvette Cedex, France, 1990, pp. 976-8.

[2] G. R. Swain, R. Kandarian, H. A. Thiessen, R. L. Poirier, and W. R. Smythe, "Progress on a Prototype Main Ring RF Cavity," in Proceedings of the 1989 IEEE Particle Accelerator Conference, Edited by Floyd Bennett and Joyce Kopta, IEEE Press, 1989 , pp. 177-179.

[3] K. Halbach and R. F. Holsinger, "SUPERFISH, a Computer Program for the Evaluation of RF Cavities with Cylindrical Symmetries," Part. Accel. vol. 7, pp. 213-22, 1976.

[4] L. S. Walling, D. E. McMurry, D. V. Neuffer, and H. A. Thiessen, "Transmission-line Impedance Measurements for an Advanced Hadron Facility," Nucl. Instrum. Methods, vol. A281, pp. 433-447, 1989. 